unity between the categories of space and time that was suggested by Einstein's work. A universe of determinate relations, in which everything proceeds according to known laws, is one which may be surveyed as a great whole in which past, present, and future are one. It is a fourdimensional unity, and for Einstein any plane section through it could be the present. The history of a particle is a simple chain of points in that whole, a "world-line."

Now events moved fast. Minkowski gave the notion of a four-dimension universe. Riemann had initiated a method of geometry in any number of dimensions; Einstein had glimpsed the possibility that, taking gravitation into account, light might be subject to acceleration, which, being interpreted in Minkowski's fashion, meant that the ordinary Euclidean expression for the element of length in a four-dimension space must be generalised. This made the work of Riemann not only useful, but also prophetic. In $r 853$ he had written that "the ground of the measure relations existing in the universe, if continuous, must consist in the binding forces acting upon it." This really implies the whole relativity doctrine; it asserts that the measure relations of the pheno- mena perceived in the universe are incapable of determination on any absolute scale, independent of the phenomena themselves. Sixty years later Einstein perceives that the gravitational field must be included among those binding forces, and must affect profoundly the measure relations in every physical aspect. Having foreseen this, Riemann had proceeded to develop a non-Euclidean geometry in any number of dimensions, and thus the germ of the calculus that. Einstein needed was created. With what success it was wielded is now well known.

Not so well known is the more recent work of Weyl. Einstein finds in universal gravitation the ground of the measure relations of the universe. But equally universal is the fact of electricity, and this universal "binding force" must equally take a part in those relations. The acuteness of Riemann's vision is doubly emphasised when we hear that Weyl discovers a further generalisation of his geometrical method which provides for electricity a place as natural and convincing as that taken by gravitation in Einstein's theory. But that is history yet in the making, and this article seeks only to traverse the course of history already complete.

\title{
Relativity and the Eclipse Observations of May, I9I9.
}

\section{By Sir Frank Dyson, F.R.S.}

I $\mathrm{N}$ I9I5 Prof. Einstein predicted, as a consequence of the generalised theory of relativity, that a ray of light from a star would be bent in its passage through the sun's gravitational field. The amount of this deflection he gave as $\mathrm{I} \cdot 75^{\prime \prime}(a / r)$, where $a$ is the sun's radius, and $r$ the nearest distance of the ray to the sun's centre. As a ray of light reaches us in the direction of the tangent to its path, the apparent position of a star, photographed during an eclipse of the sun, should therefore be displaced by an angle $1 \cdot 75^{\prime \prime}(a / r)$ outwards from the sun's centre. The field of stars surrounding the sun should thus be distorted in a definite manner by an amount within the range of accurate astronomical observation. In Fig. I, if $a, b, c$ are the positions of the stars as seen at ordinary times, and if $\mathrm{S}$ is the centre of the sun during an eclipse, then the positions in which the stars are seen or photographed during an eclipse will be $\mathrm{A}, \mathrm{B}, \mathrm{C}$. If $a$ were at a distance $3 \mathrm{O}^{\prime}$ from the sun's centre the displacement $a \mathrm{~A}$ would be $0.87^{\prime \prime}$, and if B were at a distance $9 \mathrm{o}^{\prime}$ from the sun's centre its displacement would be $0 \cdot 27^{\prime \prime}$. These are, roughly speaking, the greatest and least displacements which can be obtained in practice. Nearer than $30^{\prime}$ from the sun's centre a star's image is liable to be drowned in the corona. At greater distances than $90^{\prime}$ good images can scarcely be obtained with a simple object glass, while a doublet or other combination introduces some difficulties.

These displacements, though small, are ten times as large as those met with in determinaNo. 2677 , VOL. IO6] tions of stellar parallax, and are determined in a precisely similar manner by comparison of photographs taken at different epochs. The field of stars photographed during the eclipse must be compared with the same field photographed when the sun is in another part of the sky. Experience shows that telescopes of as long focal length as practicable should be used, and that the eclipse field and comparison field should be phorographed under as nearly as possible identical con-

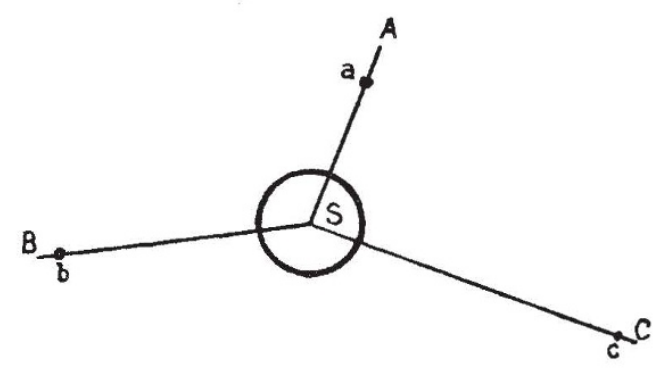

FIG. 1.

ditions as regards both the instruments themselves and the position of the field in the sky. With these precautions there was every reason to suppose that, given favourable weather, successful results would be obtained.

In the short time available for preparation it was not possible to arrange for the telescopes to be mounted equatorially, and the field of stars was reflected into fixed telescopes by cœlostat 
mirrors. The possibility had to be faced that the mirrors might suffer some distortion from the sun's heat. Apart from this, two of the mirrors did to some extent spoil the sharpness of the stellar images.

Three series of photographs were taken. Prof. Eddington and Mr. Cottingham at Principe had very cloudy weather, but obtained photographs showing a few stars. The check field obtained on two nights shortly after the eclipse gave images similar to those on the plates taken during the eclipse. The temperature during the eclipse was $77^{\circ} \mathrm{F}$., and $76.5^{\circ} \mathrm{F}$. when the check fields were taken. There was no reason to suppose any change of scale, and on this assumption the plates when measured gave for the deflection at the sun's $\operatorname{limb} \mathbf{r} \cdot 6 \mathrm{x}^{\prime \prime} \pm 0.30^{\prime \prime}$.

Dr. Crommelin and Mr. Davidson in Brazil were favoured with ideal weather conditions. They found, however, that the images on the eclipse plate differed from those taken the previous night, and from those taken on the same field of stars two months later. This is attributed to the effect of the sun's heat in distorting the colostat mirror. If it is assumed that the scale has changed, then the Einstein deflection from the series of plates is $0.90^{\prime \prime}$; if it is assumed that no real change of focus occurred, but merely a blurring of the images, the result is $\mathrm{x} \cdot 5^{6 / l}$; little weight is, however, attached to this series of photographs.

With a smaller lens of 4 -in. aperture and I9-ft. focus the same observers were extremely successful. The images taken during' the eclipse are in sharp focus and exactly similar to those on the comparison field. The result of measurement gave $1.98^{\prime \prime} \pm 0.12$ " for the deflection at the limb; seven stars were photographed, and the individual displacements, both in right ascension and in declination, were in good accordance with the law $r \cdot 75^{\prime \prime}(a / r)$. It has been pointed out by Prof. H. N. Russell that the photographs show a difference of scale of one part in 12,000 in the horizontal and vertical directions of the cœlostat mirror, and that if allowance be made for this the results for individual stars will be in still closer accordance.

The result of the eclipse, particularly of the second series of photographs at Sobral, is a close verification of Einstein's predicted displacements $1 \cdot 75^{\prime \prime}(a / r)$ radial from the sun's centre.

We proceed to consider the objections raised against this result. It has been suggested that allowance was not made correctly for the ordinary terrestrial refraction. The method adopted was that usually employed in astronomical photography, the second order terms being omitted, as they in no case amount to more than 0.02 ". The possibility of distortion of the film of the photographs, owing to the presence of the corona, has also been suggested. Examination of the plate in Phil. Trans., vol. ccxx., will show how different the conditions are from those cases in which distortion of the film has been observed. The possible displacements on the eclipse photographs could not amount to $0.05^{\prime \prime}$ for any of the stars, and would be in the wrong direction.

Prof. Anderson made the interesting suggestion that a possible error might arise from the fall of temperature in the air as the moon's shadow moves over the place of observation, thus causing an exceptional refraction. It has been shown by Prof. Eddington and Sir Arthur Schuster (NATuRE, vol. civ., pp. 372,468 ) that this effect would in all cases be very much smaller than the quantities in question. Further, it happens that at Sobral, owing to cloud in the early morning, which checked the normal daily rise of temperature, the fall during the eclipse was not more than ${ }_{2}^{\circ}$ or $3^{\circ} \mathrm{F}$.

The possibility that the observed displacements may be due to refraction by gaseous matter surrounding the sun has received a good deal of consideration. If such an atmosphere is controlled entirely by the sun's gravitation, an impossibly high density is required. Prof. Newall has investigated the consequences of assuming that gravitation is partly balanced near the sun by electrical forces and radiation pressure assumed to vary as the inverse square of the distance from the sun's centre. To obtain agreement with the eclipse observations, he finds it necessary that 0.999 of the weight of the gas should be thus balanced. The difficulties of such a supposition have been pointed out by Prof. Lindemann $(\mathrm{Ob}$ servatory, April, 1920). Further, Dr. Crommelin has directed attention to the fact that several comets have approached nearer the sun than paths of the rays of some of the stars photographed at the eclipse, yet their motion has not been retarded, or their substance entirely vaporised, although they were for two hours at this small distance from the sun and moving with a velocity of 300 kilometres a second.

\title{
Relativity and the Motion of Mercury's Perihelion.
}

\author{
By Dr. A. C. D. Crommelin.
}

THE effect of Einstein's law in changing the position of Mercury's perihelion is sometimes looked on as something mysterious and recondite; but in reality anything that disturbs the law of inverse squares in the smallest degree is bound to produce such a shift; and as in all the No. 2677 , voL. IO6] systems known to us such disturbing factors exist, the line of apses is invariably in motion.

It is easy to show that when the central force falls off more rapidly than the ratio of inverse squares, the apse-line advances; for we may consider the inverse square law as holding, with the 